\title{
Head-to-head comparison between FOLFIRINOX and gemcitabine plus nab-paclitaxel in the neoadjuvant chemotherapy of localized pancreatic cancer: a systematic review and meta-analysis
}

\author{
Rong Tang ${ }^{1,2,3,4 \#}$, Qingcai Meng ${ }^{1,2,3,4 \#}$, Wei Wang ${ }^{1,2,3,4 \#}$, Chen Liang ${ }^{1,2,3,4}$, Jie Hua ${ }^{1,2,3,4}$, Jin $\mathrm{Xu}^{1,2,3,4}$, \\ Xianjun $\mathrm{Yu}^{1,2,3,4}$, Si Shi ${ }^{1,2,3,4}$ \\ ${ }^{1}$ Department of Pancreatic Surgery, Fudan University Shanghai Cancer Center, Shanghai, China; ${ }^{2}$ Department of Oncology, Shanghai Medical \\ College, Fudan University, Shanghai, China; ${ }^{3}$ Shanghai Pancreatic Cancer Institute, Shanghai, China; ${ }^{4}$ Pancreatic Cancer Institute, Fudan University, \\ Shanghai, China \\ Contributions: (I) Conception and design: X Yu, S Shi, J Xu; (II) Administrative support: J Hua, C Liang; (III) Provision of study materials or patients: \\ W Wang, Q Meng; (IV) Collection and assembly of data: W Wang, Q Meng; (V) Data analysis and interpretation: R Tang; (VI) Manuscript writing: \\ All authors; (VII) Final approval of manuscript: All authors. \\ "These authors contributed equally to this work. \\ Correspondence to: Si Shi. Department of Pancreatic Surgery, Fudan University Shanghai Cancer Center, No. 270 Dong'An Road, Shanghai \\ 200032, China. Email: shisi@fudanpci.org; Xianjun Yu. Shanghai Pancreatic Cancer Institute, No. 270 Dong’An Road, Shanghai 200032, China. \\ Email: yuxianjun@fudanpci.org.
}

Background: The benefits of neoadjuvant chemotherapy (NCT) in pancreatic cancer (PC) have been realized and gradually accepted. FOLFIRINOX and gemcitabine and nab-paclitaxel (GA) are the two most widely used regimens for PC NCT.

Methods: The literature was systematically reviewed by searching MEDLINE, EMBASE, Web of Science and the Cochrane Library for studies published until September 2020.

Results: Eight studies were eligible for the meta-analysis. Compared to GA, neoadjuvant FOLFIRINOX significantly prolonged overall survival [hazard ratio $(\mathrm{HR})=0.65,95 \%$ confidence interval $(95 \% \mathrm{CI})$ : 0.55-0.77; $\mathrm{P}<0.001]$. FOLFIRINOX provided better survival benefits in the first three years after surgery; however, the 4- and 5-year survival probabilities of the two strategies were similar based on a conservative estimation in the random effect model. The perioperative parameters analysed included perineural invasion (PNI), lymphovascular invasion (LVSI), R0 status, postoperative complications and resection rate. The PNI rate was marginally elevated in the GA group compared with the FOLFIRINOX cohort [79.8\% vs. 70.5\%, odds ratio $(\mathrm{OR})=0.70,95 \% \mathrm{CI}: 0.47-1.06, \mathrm{P}=0.09$ ], which may account for the potential survival benefits of FOLFIRINOX.

Conclusions: The results of our meta-analysis suggest that FOLFIRINOX is non-inferior to GA in patients who are FOLFIRINOCX capable.

Keywords: Neoadjuvant chemotherapy (NCT); FOLFIRINOX; nab-paclitaxel; pancreatic cancer (PC); metaanalysis

Submitted Jan 08, 2021. Accepted for publication Mar 19, 2021.

doi: $10.21037 / \mathrm{gs}-21-16$

View this article at: http://dx.doi.org/10.21037/gs-21-16 


\section{Introduction}

Pancreatic cancer (PC) is one of the most lethal malignancies, with an incidence rate that equals its mortality rate (1). The incidence of PC has been steadily increasing over recent decades, and it is projected to become the second leading cause of cancer-related death by 2030 (2). However, the treatment options for PC are limited. Surgical resection is currently the only approach for the radical treatment of $\mathrm{PC}$, while curative resection of PC occurs in only $10-15 \%$ of patients (3). Hence, how to turn unresectable cases into resectable ones is of great importance to the treatment of PC. Neoadjuvant chemotherapy (NCT) is an effective weapon that enables the radical resection of borderline resectable (BRPC) and locally advanced PC (LAPC) and eradicates occult cancer (4). Many clinical trials have shown the survival benefits of NCT over surgery as the first line of treatment in BR and LA cases (4-6). Some studies have even shown that neoadjuvant therapy may offer survival benefits over upfront surgery in resectable PC (RPC) despite controversies (7-11). In addition, NCT could also identify poor responders who progress on treatment preoperatively, sparing them from futile surgery (12). Overall, NCT seems to be a major trend for optimizing the survival outcome of localized PC.

Nonetheless, which neoadjuvant regimen harbours the utmost clinical benefits remains controversial due to a lack of evidence. Both oxaliplatin, irinotecan, fluorouracil, and leucovorin (FOLFIRINOX) and gemcitabine plus nab-paclitaxel (GA) are suggested by clinical practice guidelines as first-line therapies for patients with BRPC and LAPC, given data from two phase III clinical trials that demonstrated longer overall survival in metastatic PC patients treated with either FOLFIRINOX or GA than in those treated with gemcitabine alone $(13,14)$. Currently, although several studies have compared the efficacy of FOLFIRINOX and GA in localized PC, no consensus has been reached (15-22).

Some studies reported that FOLFIRINOX prolongs survival more than GA $(19,21,22)$, while others showed that the efficacy of these two regimens is similar (16-18,20,23).

In this context, we conducted a systematic review and meta-analysis of published data to determine the difference in clinical outcomes brought by preoperative use of FOLFIRINOX and GA. Our aim was to determine the optimal regimen for NCT in localized PC.

\section{Methods}

The systematic review was performed according to the MOOSE guidelines and reporting checklist (available at http://dx.doi.org/10.21037/gs-21-16) (24). Study selection was performed by two independent authors (RT and QM) by reviewing the title and abstract followed by full-text screening, where primary and secondary outcomes were extracted. Disagreements were resolved by team discussion.

\section{Search strategy}

The literature was systematically reviewed by searching MEDLINE, EMBASE, Web of Science and the Cochrane Library for studies published until 11 September 2020. We designed a broad retrieval strategy to include as many relevant studies as possible in case of some unexpected lack of important data. The full retrieval terms are listed as follows: "MEDLINE: ((pancreatic cancer[title/ abstract] OR PDAC[title/abstract] OR pancreatic ductal adenocarcinoma[title/abstract]) AND (neoadjuvant)) AND (FOLFIRINOX OR gemcitabine OR FFX OR GNP OR GA OR nabpaclitaxel OR nab-paclitaxel OR abraxane OR Taxol); EMBASE: ('PANCREATIC CANCER': abstract (ab), title (ti) OR PDAC: ab, ti OR 'pancreatic ductal adenocarcinoma': ab, ti) AND neoadjuvant AND (gemcitabine OR FOLFIRINOX OR FFX OR GA OR GNP OR nabpaclitaxel OR 'nab paclitaxel' OR abraxane OR Taxol); Web of Science: topic: (PANCREATIC CANCER OR PDAC OR pancreatic ductal adenocarcinoma) AND all fields: (neoadjuvant) AND all fields: (FOLFIRINOX OR gemcitabine OR FFX OR GNP OR GA OR nabpaclitaxel OR nab-paclitaxel OR abraxane OR Taxol); Cochrane: (PANCREATIC CANCER OR PDAC OR pancreatic ductal adenocarcinoma): ti, ab, kw AND (neoadjuvant) AND (FOLFIRINOX OR gemcitabine OR FFX OR GNP OR GA OR nabpaclitaxel OR nabpaclitaxel OR abraxane OR Taxol)" (word variations have been searched).

\section{Study selection and data extraction}

Studies were included in the present meta-analysis if they met the following criteria: (I) Studies involved localized PC, including RPC, BRPC and LAPC. (II) Patients preoperatively received FOLFIRINOX and GA intervention and were divided into two independent cohorts. 
(III) Studies compared the efficacy of FOLFIRINOX and GA to overall survival in patients with localized PC. (IV) Studies contained survival curves or other forms of outcome exhibition in the full text, making it possible to extract data.

Studies were excluded from the present meta-analysis if they met the following criteria: (I) Studies focused on metastatic PC. (II) Patients preoperatively received regimens other than FOLFIRINOX or GA or patients postoperatively received FOLFIRINOX or GA without neoadjuvant treatment. (III) Studies lacked adequate data to compare the difference in efficacy between FOLFIRINOX and GA in terms of the overall survival of PC. (IV) Studies were review articles, notes, case reports or animal studies.

The primary outcome was the overall survival of patients. The secondary outcomes were resection rate, R0 status, perineural invasion (PNI) rate, lymphovascular invasion (LVSI) rate, postoperative complications and 1- to 5 -year survival rate. If the study provided data adjusted for confounding factors, then we extracted the adjusted data for the following meta-analysis.

\section{Quality assessment}

The quality assessment of observational studies was suggested to be done with the Newcastle-Ottawa Scale (NOS) (25). Two independent authors gave a score to each study based on their evaluation of the three sections of this scale (selection, comparability and outcome). Discrepancies were resolved by discussion among authors.

\section{Outcome measures and statistical analysis}

Hazard ratios (HRs) and 95\% confidence intervals (CIs) were used as indicators of the difference in efficacy between FOLFIRINOX and GA in terms of the overall survival of patients with localized PC. Odds ratios (ORs) were computed to assess the differences in 1- to 5-year survival and perioperative parameters between the FOLFIRINOX and GA cohorts. Heterogeneity of the studies was explored using Cochrane's Q test of heterogeneity and $\mathrm{I}^{2}$ statistic. If $\mathrm{I}^{2}>25 \%$ and $\mathrm{P}<0.05$ in the heterogeneity test, we performed sensitivity analysis (leave-one-out analysis) to assess whether the obvious heterogeneity came from a specific study. If so, we further evaluated the methodological heterogeneity between this study and its counterparts and excluded it when necessary. In most cases, sensitivity analysis does not distinguish the culprit contributing to significant heterogeneity. In this case, we applied the random effect model to compute the pooled effect value with a conservatively extended CI. For the subgroup with $\mathrm{I}^{2}<25 \%$, we applied the fixed effect model to compute the pooled effect value. Publication bias was assessed by the Begg's test, and the symmetry of the funnel plot was assessed using Stata 15.1. Given the insufficient number of included studies in other models, we only assessed publication bias in the model for overall survival.

The pooled HR for overall survival was computed in Stata 15.1, while the pooled ORs for the 1- to 5-year survival rate and perioperative parameters were computed in Revman 5.4. $\mathrm{P}<0.05$ was regarded as indicating statistical significance in this study.

\section{Results}

\section{Literature search}

The systematic search yielded 3,251 records from EMBASE, Web of Science, MEDLINE, and the Cochrane Library. Then, 2,453 records were retained after duplicates were removed. According to the title and abstract, 2,388 articles were regarded as not relevant to our study design and hence were discarded. Next, the remaining 65 articles were retrieved for full-text screening, among which 57 studies were excluded based on our criteria. Specifically, one study was only a clinical trial protocol without a follow-up report; 9 studies reported a gemcitabine-based treatment, while they did not specify the number/proportion and/or follow-up data of patients who received GA; 26 studies did not have a neoadjuvant-intended design; 21 studies were focused only on metastatic diseases. Finally, 8 studies were eligible for the meta-analysis of overall survival. In addition, each study harboured at least one secondary endpoint for quantitative synthesis. In detail, the 1- to 3-year survival rates were extracted from 8 studies; the 4-year survival rate was extracted from 5 studies; the 5 -year survival rate was extracted from 4 studies; the PNI rate was extracted from 4 studies; the LVSI rate was extracted from 4 studies; the resection rate was extracted from 4 studies; and the postoperative complication rate was extracted from 3 studies. The search results and selection process are summarized in a flowchart (Figure 1).

\section{Study characteristics}

All eight included studies were retrospective (Table 1). Among them, six studies were carried out in the United 


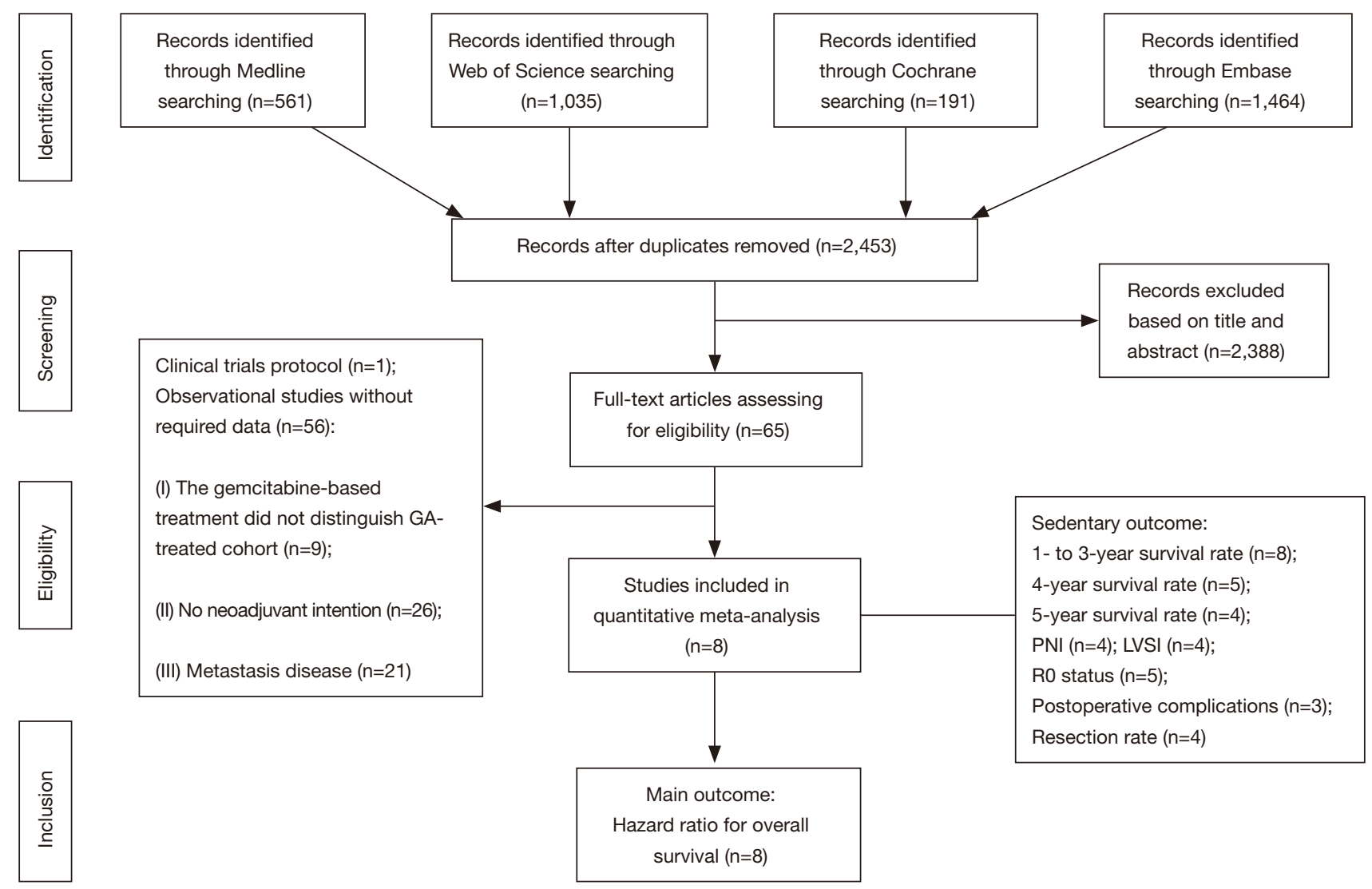

Figure 1 Flow chart summarizing the procedures of this meta-analysis.

States, while the others came from Italy and Germany. Notably, these studies were reported very recently from 2018 to 2020 , of which two studies were multi-centre studies while the others were single-centre studies. The number of patients in the included studies varied from 56 to 280 . The aggregated numbers of patients preoperatively treated with FOLFIRINOX versus GA were 746 and 611, respectively. The proportions of patients with different resectability in two groups were summarized as Table S1. Besides, the percentage of patients with different lymph nodes status were also presented in Table S2. Hence, this meta-analysis could provide compelling evidence in view of a large sample size. In addition, the majority of studies declared that the decision for NCT was determined by multidisciplinary discussion, which ensured the reasonability of patient inclusion.

The NOS was applied to evaluate the quality of the included studies. The score of each study varied from 5 to 8 , which means that the overall design of these studies was acceptable despite some flaws. For example, most studies did not maintain the comparability of age between FOLFIRINOX and GA groups. This intrinsic selective bias was attributed to the toxic effect of FOLFIRINOX being more robust than that of GA. Given that, clinicians were inclined to subjectively prescribe FOLFIRINOX to younger patients. Although some studies adjusted for several confounding factors when they evaluated the efficacy of FOLFIRINOX versus GA in terms of overall survival in the following regression model $(16,19,20)$, we still assessed the quality based on their primary design.

\section{Effects of FOLFIRINOX versus $G A$ on overall survival}

Three of our included studies (3/8) demonstrated a significantly prolonged overall survival in FOLFIRINOXtreated patients, while the others declared that the efficacy of these two regimens was similar. In this meta-analysis and overall, compared to GA, neoadjuvant FOLFIRINOX was associated with prolonged overall survival ( $\mathrm{HR}=0.65,95 \%$ CI: $0.55-0.77, \mathrm{P}<0.001$ ) (Figure $2 A$ ). The subgroup analysis 
Table 1 Characteristics of all studies included in the meta-analysis for quantitative synthesis

\begin{tabular}{|c|c|c|c|c|c|c|c|}
\hline Study & Resectability & Region & $\begin{array}{c}\text { Multi- or } \\
\text { single-centre }\end{array}$ & $\begin{array}{l}\text { FOLFIRINOX- } \\
\text { treated patients }\end{array}$ & $\begin{array}{l}\text { GA-treated } \\
\text { patients }\end{array}$ & $\begin{array}{l}\text { Decision for } \\
\text { neoadjuvant }\end{array}$ & NOS score \\
\hline $\begin{array}{l}\text { Dhir et al., } \\
2018\end{array}$ & $\mathrm{RPC}$ and BRPC & United States & Single-centre & 73 & 120 & $\begin{array}{l}\text { Multidisciplinary } \\
\text { tumour board }\end{array}$ & 7 \\
\hline $\begin{array}{l}\text { Chapman } \\
\text { et al., } 2018\end{array}$ & BRPC and LAPC & United States & Single-centre & 55 & 12 & $\begin{array}{c}\text { Pancreatic } \\
\text { and biliary } \\
\text { multidisciplinary } \\
\text { team }\end{array}$ & 7 \\
\hline $\begin{array}{l}\text { Perri et al., } \\
2020\end{array}$ & $\begin{array}{c}\text { RPC, BRPC and } \\
\text { LAPC }\end{array}$ & United States & Single-centre & 140 & 140 & NA & 8 \\
\hline $\begin{array}{l}\text { Wolfe et al., } \\
2020\end{array}$ & BRPC or LAPC & United States & Single-centre & 52 & 20 & $\begin{array}{c}\text { Multidisciplinary } \\
\text { pancreatic oncology } \\
\text { team }\end{array}$ & 6 \\
\hline $\begin{array}{l}\text { Napolitano } \\
\text { et al., } 2019\end{array}$ & LAPC & Europe (Italy) & Single-centre & 35 & 21 & $\begin{array}{l}\text { Multidisciplinary } \\
\text { teamwork }\end{array}$ & 6 \\
\hline $\begin{array}{l}\text { Weniger et al., } \\
2020\end{array}$ & LAPC and BRPC & Europe (Germany) & Multi-centre & 103 & 32 & $\begin{array}{l}\text { Multi-disciplinary } \\
\text { decision }\end{array}$ & 6 \\
\hline $\begin{array}{l}\text { Gage et al., } \\
2019\end{array}$ & $N A^{*}$ & United States & Single-centre & 105 & 175 & NA & 5 \\
\hline
\end{tabular}

*, the authors declared in the "Methods" section that the patients they enrolled underwent neoadjuvant chemotherapy followed by Whipple procedures (the proportions of each type were not presented). GA, gemcitabine and nab-paclitaxel; RPC, resectable pancreatic cancer; BRPC, borderline resectable pancreatic cancer; LAPC, locally advanced pancreatic cancer; NOS, Newcastle-Ottawa Scale.

included patients who received NCT followed by surgical resection and yielded a similar conclusion ( $\mathrm{HR}=0.60,95 \%$ CI: $0.47-0.77, \mathrm{P}<0.001$ ) (Figure $2 B$ ). Given that patients treated with FOLFIRINOX were universally younger than those treated with GA, we performed a subgroup analysis including studies adjusted for age (Figure S1). The results showed that compared with GA, FOLFIRINOX was still beneficial in terms of prolonged overall survival. No obvious heterogeneity was detected in this subgroup $\left(\mathrm{I}^{2}=0.0 \%, \mathrm{P}=0.84\right)$. Hence, preoperative treatment with FOLFIRINOX was associated with better survival than GA.

No obvious heterogeneity was detected statistically in the abovementioned two models $\left(\mathrm{I}^{2}=0.0 \%, \mathrm{P}=0.809\right.$ and 0.994 , respectively), which was the reason why we applied a fixed effect model to pool the HR. We also performed the Begg's test and depicted a funnel plot to assess publication bias (Figure S2). Although the Begg's test did not show significant publication bias ( $\mathrm{P}=0.174$, continuity corrected), the funnel plot visually showed asymmetry, which may be attributed to the insufficient number of included studies.

\section{Effects of FOLFIRINOX versus GA on 1- to 5-year survival rate}

We further investigated the influence of FOLFIRINOX and GA on the 1- to 5-year survival rate in patients with PC. The pooled 1-year survival rate was higher in the FOLFIRINOX group than in the GA group $(88.4 \%$ and $77.3 \%$, respectively; $\mathrm{OR}=2.19,95 \%$ CI: $1.16-4.16, \mathrm{P}=0.02$ ) (Figure 3A). Similarly, the pooled 2- and 3-year survival rates were also significantly increased in the FOLFIRINOX group relative to the GA group (OR $=1.66,95 \%$ CI: $1.13-$ 2.42, $\mathrm{P}=0.01$ ) and $\mathrm{OR}=1.82,95 \% \mathrm{CI}: 1.19-2.78, \mathrm{P}=0.006$ ), respectively) (Figure 3B,C). Intermediate heterogeneity was observed in these models $\left(\mathrm{I}^{2}=51 \%, 40 \%\right.$ and $45 \%$ for 1 -, 2 and 3-year survival rates, respectively); hence, we applied a random effect model to calculate the pooled OR to avoid false positive events and compute a conservative result. Only five studies harboured a 4-year survival rate and could be included in the meta-analysis. FOLFIRINOX promoted the 4-year survival rate of patients with $\mathrm{PC}$ relative to that of those with GA only in the fixed effect model $(\mathrm{OR}=1.54$, 


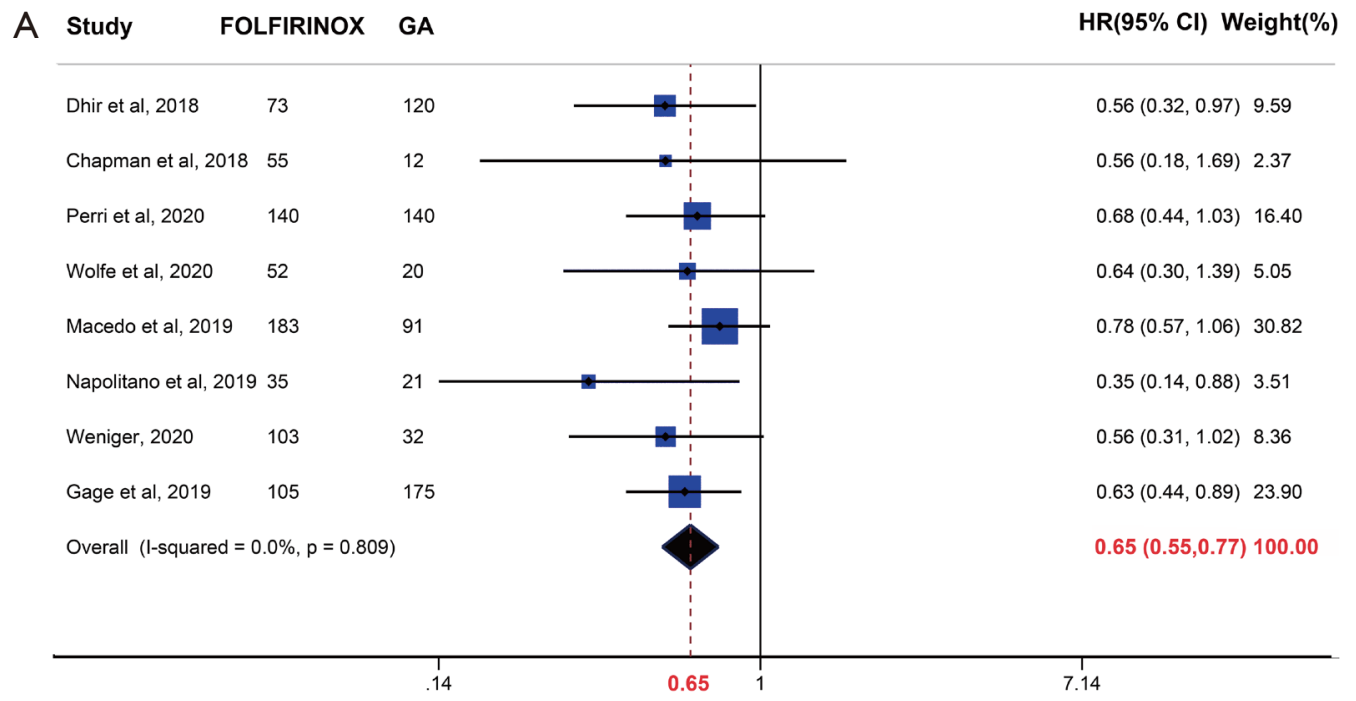

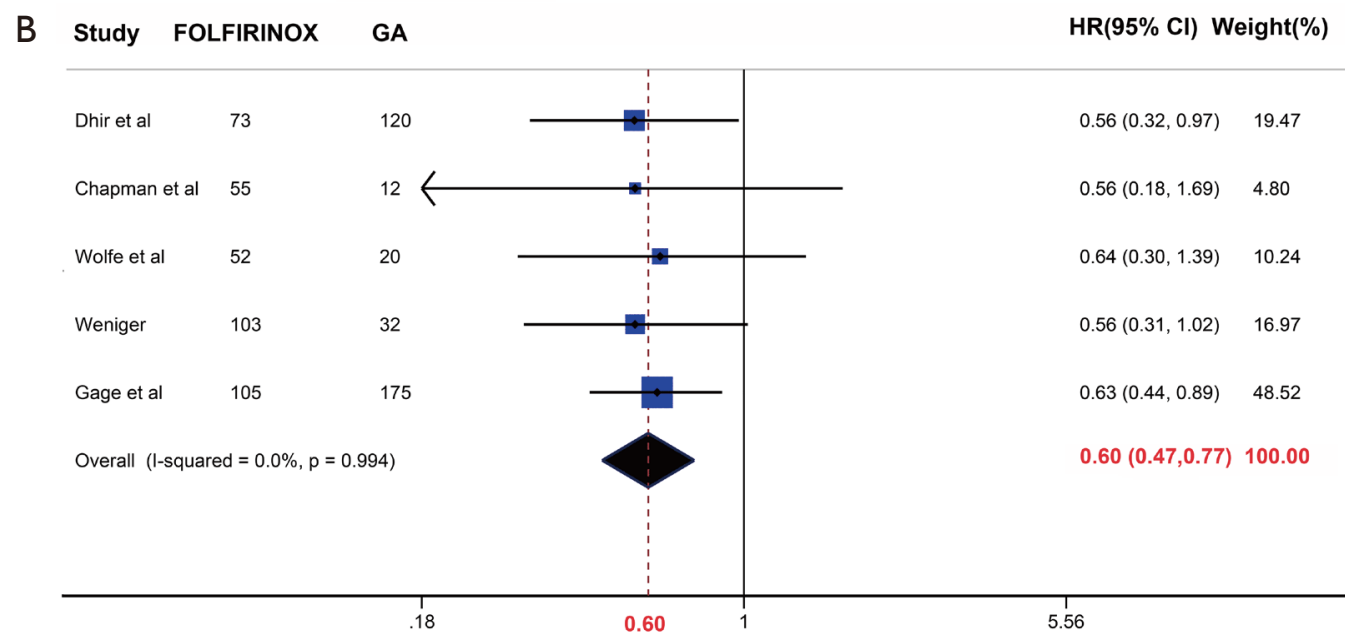

Figure 2 Forrest plot showing the pooled hazard ratio (HR) for the overall survival interval of patients who received preoperative FOLFIRINOX versus gemcitabine and nab-paclitaxel (GA). (A) Neoadjuvant chemotherapy (NCT) with/without surgery. (B) NCT followed by surgery.

95\% CI: 1.14-2.08, $\mathrm{P}=0.005$ ) (Figure S3A). However, when obvious heterogeneity was observed $\left(\mathrm{I}^{2}=69 \%, \mathrm{P}=0.01\right)$, we accordingly adjusted the model to a random effect model and found that the statistical significance was compromised (OR $=1.36,95 \%$ CI: 0.74-2.48) (Figure S3B). Regarding the five-year survival rate, only four studies were included in the meta-analysis. Similarly, we detected obvious heterogeneity in this model $\left(\mathrm{I}^{2}=68 \%\right)$ and decided to use a random effect model to compute the pooled OR (Figure S3B). With the limited sample size, we conservatively concluded that the efficacy of FOLFIRINOX and GA in terms of the 5-year survival rate was similar $(\mathrm{OR}=1.58,95 \% \mathrm{CI}$ : $0.82-3.04$,
$\mathrm{P}=0.18$ ), although FOLFIRINOX was more effective in the fixed effect model (OR $=1.51,95 \%$ CI: $1.09-2.09, \mathrm{P}=0.03$ ) (Figure S3A).

\section{Effects of FOLFIRINOX versus $G A$ on perioperative parameters}

The perioperative parameters included in this metaanalysis comprised PNI, LVSI, R0 status, postoperative complications and resection rate. Each subgroup consisted of at least 3 studies, and their sample size varied from 317 to 518 . The pooled resection rate was statistically 
A

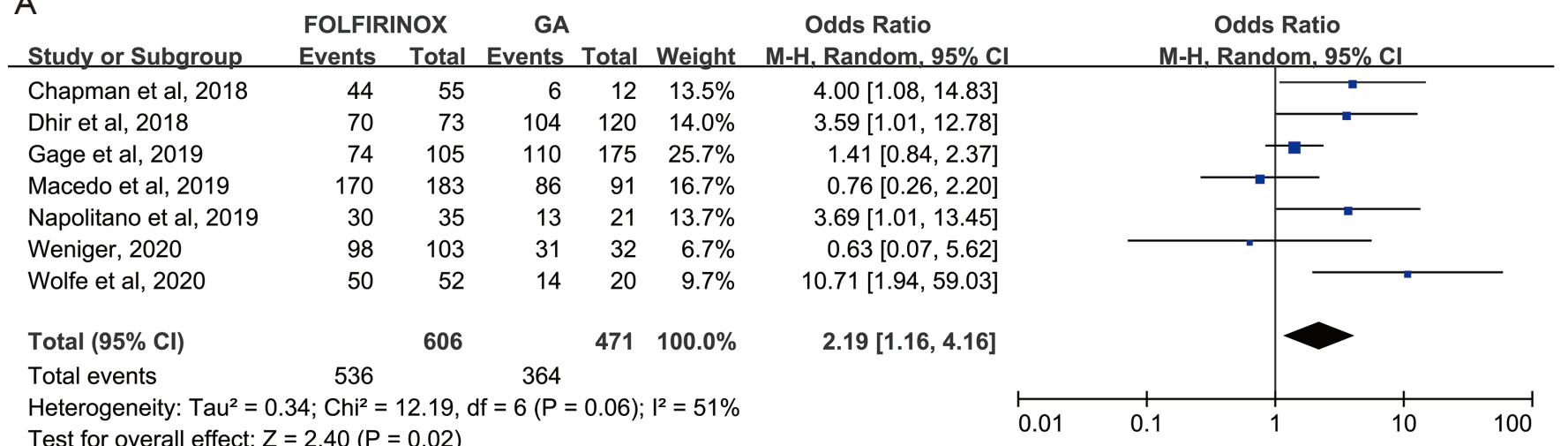

B

Study or Subgroup

FOLFIRINOX

GA

Odds Ratio Events Total Events Total Weight M-H, Random, 95\% Cl

Chapman et al, 2018

$\begin{array}{lllll}12 & 55 & 2 & 12 & 4.7 \%\end{array}$

Dhir et al, 2018

$\begin{array}{lllll}49 & 73 & 81 & 120 & 19.1 \%\end{array}$

Gage et al, 2019

$57 \quad 105$

$\begin{array}{lll}56 & 175 & 23.2 \%\end{array}$

Macedo et al, 2019

$127 \quad 183$

$61 \quad 91 \quad 21.8 \%$

Napolitano et al, 2019

17
74

Weniger, 2020

Wolfe et al, 2020

$74 \quad 103$

$\begin{array}{lll}4 & 21 & 7.3 \%\end{array}$

$\begin{array}{lll}17 & 32 & 13.9 \%\end{array}$

$\begin{array}{lllll}31 & 52 & 10 & 20 & 10.0 \%\end{array}$

Total $(95 \% \mathrm{Cl})$

606

$471 \quad 100.0 \%$

Total events $367 \quad 231$

Heterogeneity: $\mathrm{Tau}^{2}=0.10 ; \mathrm{Chi}^{2}=10.01, \mathrm{df}=6(\mathrm{P}=0.12) ; \mathrm{I}^{2}=40 \%$

Test for overall effect: $Z=2.59(P=0.010)$

C

FOLFIRINOX

GA

$1.40[0.27,7.25]$
$0.98[0.53,1.83]$
$2.52[1.53,4.15]$
$1.12[0.65,1.91]$
$4.01[1.12,14.37]$
$2.25[1.00,5.09]$
$1.48[0.52,4.16]$
$1.66[1.13,2.42]$

$1.40[0.27,7.25]$

$0.98[0.53,1.83]$

$2.52[1.53,4.15]$

$1.12[0.65,1.91]$

$.01[1.12,14.37]$

$2.25[1.00,5.09]$

$1.66[1.13,2.42]$

Odds Ratio

Study or Subgroup Events Total Events Total Weight

Chapman et al, 2018

$\begin{array}{lllll}3 & 55 & 2 & 12 & 4.3 \%\end{array}$

Dhir et al, 2018

Gage et al, 2019

$\begin{array}{lllll}39 & 73 & 45 & 120 & 21.2 \%\end{array}$

Macedo et al, 2019

$\begin{array}{lllll}40 & 105 & 32 & 175 & 22.5 \%\end{array}$

Napolitano et al, 2019

$90 \quad 183$

$37 \quad 91 \quad 23.7 \%$

Weniger, 2020

$15 \quad 35$

$\begin{array}{lll}0 & 21 & 2.0 \%\end{array}$

Wolfe et al, 2020

$52 \quad 103$

$13 \quad 32 \quad 15.7 \%$

Total $(95 \% \mathrm{Cl})$

$24 \quad 52$

$6 \quad 20 \quad 10.5 \%$

Total events

606

$471 \quad 100.0 \%$

Heterogeneity: $\mathrm{Tau}^{2}=0.13 ; \mathrm{Chi}^{2}=10.95, \mathrm{df}=6(\mathrm{P}=0.09) ; \mathrm{I}^{2}=45 \%$

Test for overall effect: $Z=2.77(P=0.006)$

Odds Ratio

M-H, Random, 95\% Cl

$0.29[0.04,1.95]$

$1.91[1.06,3.45]$

$2.75[1.59,4.76]$

$1.41[0.85,2.35]$

$32.51[1.82,579.42]$

$1.49[0.67,3.33]$

$2.00[0.67,6.01]$

$1.82[1.19,2.78]$

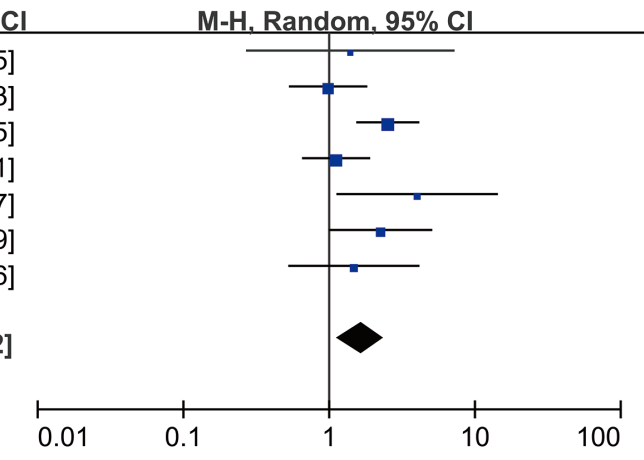

Figure 3 Forrest plot depicting the difference in 1- to 3 -year survival probability between patients who received preoperative FOLFIRINOX versus gemcitabine and nab-paclitaxel (GA).

similar between the FOLFIRINOX and GA groups (OR $=1.07,95 \%$ CI: 0.61-1.88, $\mathrm{P}=0.80$ ) (Figure 4A). Likewise, the pooled R0 status rate was comparable between FOLFIRINOX-treated patients and the GA cohort (OR $=1.19,95 \%$ CI: $0.79-1.79, \mathrm{P}=0.41$ ) (Figure 4B). In contrast, the PNI rate was marginally elevated in the GA group compared with the FOLFIRINOX cohort $(70.5 \%$ vs. $79.8 \%$, OR $=0.70,95 \%$ CI: $0.47-1.06), \mathrm{P}=0.09)$, which may account for the potential survival benefits brought by FOLFIRINOX (Figure 4C). However, there were no significant differences in LVSI rate or postoperative complication events between the FOLFIRINOX and GA groups ( $\mathrm{P}=0.43$ and 0.47 , respectively) (Figure $4 D, E$ ). Obvious heterogeneity was detected in the model for resection rate, LVSI and postoperative complications $\left(\mathrm{I}^{2}=74 \%, 65 \%\right.$ and $70 \%$, respectively). Accordingly, we 


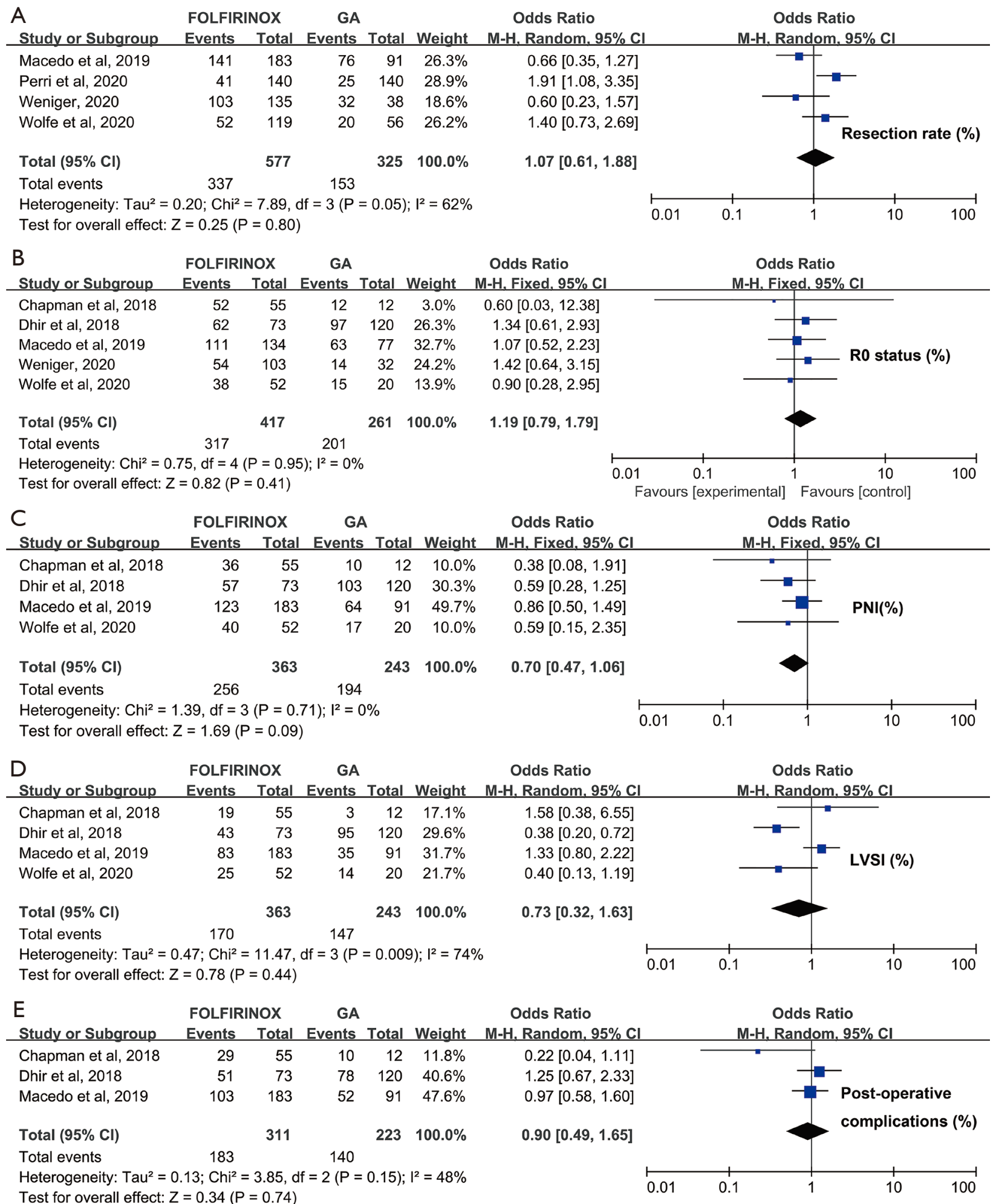

Figure 4 Quantitative comparison of perioperative parameters between patients who received preoperative FOLFIRINOX versus gemcitabine and nab-paclitaxel (GA). (A) Resection rate. (B) R0 status. (C) Perineural invasion (PNI). (D) Lymphovascular invasion (LVSI). (E) Postoperative complications. 
applied a random effect model to yield conservative conclusions. For the model of R0 status and PNI rate, we applied a fixed effect model in view of its lack of heterogeneity (both $\mathrm{I}^{2}=0.0 \%$ ).

Tumour size and CA19-9 variation were also valuable indicators for evaluating the efficacy of NCT. However, the two indicators could not be quantitatively analysed due to the absence or inconsistency in the forms of data reporting. Hence, we performed a qualitative analysis to visualize the variations in tumour size and CA19-9 after NCT in the FOLFIRINOX and GA cohorts (Figure S4). Overall, the slope that reflects that the trend of decreased tumour size was more pronounced in the FOLFIRINOX group than in the GA group. However, no obvious difference in CA19-9 variation was visually observed between the FOLFIRINOX and GA cohorts.

\section{Discussion}

The survival benefits of NCT have been universally acknowledged in most gastrointestinal cancers, supported by several randomized controlled trials (RCTs) (26-28). With the experience gained from studies of other cancers, an increased number of studies have begun to investigate the feasibility and potential benefits of NCT for localized PC $(10,29,30)$. Recently, a multi-centre, randomized, parallel-group, phase III trial by Versteijne et al. compared the prognosis of patients with localized PC receiving NCT with that of patients undergoing upfront surgery (4). Their results showed that compared with immediate surgery, NCT significantly prolonged disease-free survival and promoted the R0 resection rate. Nonetheless, this RCT did not show any benefits in terms of the overall survival of patients who received NCT. A recent meta-analysis including six RCTs demonstrated that compared with surgery first, NCT resulted in improved overall survival, increased negative lymph node counts and increased $\mathrm{R} 0$ resection rate in localized PC (31). A plausible reason for the discrepancy among these RCT studies might be the different use of regimens for NCT.

Previous RCTs have shown the superiority of FOLFIRINOX and GA compared with gemcitabine alone in the adjuvant chemotherapy of advanced PC $(13,14)$. Subsequent studies also tried to determine which is the better choice in the treatment of metastatic PC. Pusceddu et al. recently presented a meta-analysis of nonrandomized "real world" studies (32). In their study, 16 retrospective studies including 3,813 patients $(2,123$ treated with GA and 1,690 treated with FOLFIRINOX) were enrolled and demonstrated that the overall survival and progression-free survival were similar between the FOLFIRINOX and GA groups. Nonetheless, whether FOLFIRINOX improved patient prognosis relative to that with GA lacked compelling evidence and remains controversial. A retrospective, multiinstitutional study performed by Macedo et al. evaluated the prognosis of patients with PC undergoing NCT followed by curative pancreatectomy (17). In their study, the efficacy of FOLFIRINOX and GA was similar in terms of either overall survival or recurrence-free interval $(\mathrm{P}=0.804$ and $\mathrm{P}=0.618$, respectively). However, this study failed to match many confounding factors, such as chemotherapy cycle, age and radiological classification, which could undermine the accuracy of the outcome. Perri et al schemed a propensitymatched cohort including 280 patients with localized PC, in which 140 patients preoperatively received FOLFIRINOX, while the other 140 patients were treated with GA before surgical resection (16). In the multivariable regression model, FOLFIRINOX was marginally beneficial relative to GA in terms of overall survival $(\mathrm{HR}=0.68, \mathrm{P}=0.07$ ). Another recent study applied propensity-type analysis to control for confounding factors (19). The use of inverse probability weighting (IPW) estimators in propensity-type analysis has the potential to account for confounders and make it equally likely for the subjects to be assigned to each treatment arm when randomized data were absent (33). The results demonstrated that neoadjuvant FOLFIRINOX significantly improved overall survival over that with GA after adjusting for potential confounding factors. A lot of case reports also separately showed the successful conversion cases by neoadjuvant GA or FOLFIRINOX (34-40). For example, Gostimir $e t a l$. presented a case whose lesion had decreased in size and complete pathological response after underwent treatment with a total of 13 cycles of FOLFIRINOX.

Several other studies have also compared the abilities of neoadjuvant FOLFIRINOX and GA to improve patient prognosis; however, no consensus has been reached. Hence, we designed this study to systematically review the published studies that compared the efficacy of FOLFIRINOX and GA in a neoadjuvant setting. Our study illustrated that FOLFIRINOX conferred prolonged overall survival $(\mathrm{HR}=0.65, \mathrm{P}<0.05)$ and $1-, 2$ - and 3 -year survival rates $(\mathrm{OR}=2.19,1.66$ and 1.85 , respectively, $\mathrm{P}<0.05)$. Further, this survival benefit of FOLFIRINOX may be attributed to the elevated PNI rate in GA-treated patients $(\mathrm{OR}=0.70, \mathrm{P}=0.09)$. Hence, the results of this meta-analysis suggest that FOLFIRINOX should be preferred in NCT 
for localized PC.

One important subgroup analysis including studies that had confounding factors adjusted (such as age) also presented identical results (Figure S1). Heterogeneity between studies was statistically minor in most models of our study. For models with obvious heterogeneity, we performed sensitivity analysis (leave-one-out analysis) to assess whether the obvious heterogeneity came from some specific study. If so, we further evaluated the methodological heterogeneity between this study and its counterparts and excluded it when necessary. In most cases, sensitivity analysis does not distinguish the culprit for significant heterogeneity (data not shown). In those cases, we applied a random effect model to compute the pooled effect value with a conservatively extended CI.

An ongoing, well-organized RCT named SWOG1505 (NCT02562716) may confirm our results (41). Although this study was confronted with some challenges, the resection rate and major pathologic response rate were satisfactory, with $76 \%$ patients completing preoperative therapy and undergoing surgery and $33 \%$ having a complete or major pathologic response to therapy. In fact, compared to the standard of the use of single-agent gemcitabine, the use of multi-agent chemotherapy for both advanced and localized PC has been demonstrated to improve patient survival (42). Hence, given that SWOG1505 preliminarily showed satisfactory results, future clinical trials are encouraged to apply multi-agent modalities, especially FOLFIRINOX, according to our results.

The present study has some limitations. First, almost all the included studies were retrospectively designed, which may introduce some unexpected bias. Even though Perri et al used a prospectively maintained database, the authors admitted that it was still a retrospective and singleinstitution design. Second, the comparability of baseline characteristics was not well controlled in some studies. For example, patients were universally younger in the FOLFIRINOX arm than in the GA cohort. Although the unbiased subgroup analysis showed a conclusion similar to that of the whole population analysis, more prospective and baseline-matched studies are expected. Third, due to the difficulty in acquiring primary data from the included studies, some valuable perioperative events could not be quantitatively analysed, such as CA19-9 concentration and tumour volume. However, this study is the first metaanalysis to identify the best regimen for NCT of PC through head-to-head comparison with a large sample size. In addition, we incorporated several important indicators as secondary outcomes, including 1- to 5-year survival probability and some perioperative parameters, which provides useful information for clinical practice. Another issue that should be considered is that studies rarely reported the percentage of combined venous or arterial resection in groups received different neoadjuvant agents. Given the dramatic effects of combined venous or arterial resection on patients' prognosis, future studies are expected to report the comparability of vessels resection and other surgical extent.

In conclusion, the results of our meta-analysis suggest that the results of our meta-analysis suggest that FOLFIRINOX is non-inferior to GA in patients who are FOLFIRINOCX capable. Future studies are encouraged to explore the optimal dosage and number of chemotherapy cycles of FOLFIRINOX as NCT for PC.

\section{Acknowledgments}

We would like to thank the instruction from Dr. Yiyin Zhang in Zhejiang University.

Funding: This work was supported in part by the National Natural Science Foundation of China (No. 81802352, 81772555 and 81902428), the National Science Foundation for Distinguished Young Scholars of China (No. 81625016), the Shanghai Sailing Program (No. 17YF1402500), the Scientific Innovation Project of Shanghai Education Committee (2019-01-07-00-07-E00057) and the Clinical and Scientific Innovation Project of Shanghai Hospital Development Center (SHDC12018109).

\section{Footnote}

Reporting Checklist: The authors have completed the MOOSE reporting checklist. Available at http://dx.doi. org/10.21037/gs-21-16

Conflicts of Interest: All authors have completed the ICMJE uniform disclosure form (available at http://dx.doi. org/10.21037/gs-21-16). The authors have no conflicts of interest to declare.

Ethic Statement: The authors are accountable for all aspects of the work in ensuring that questions related to the accuracy or integrity of any part of the work are appropriately investigated and resolved.

Open Access Statement: This is an Open Access article distributed in accordance with the Creative Commons 
Attribution-NonCommercial-NoDerivs 4.0 International License (CC BY-NC-ND 4.0), which permits the noncommercial replication and distribution of the article with the strict proviso that no changes or edits are made and the original work is properly cited (including links to both the formal publication through the relevant DOI and the license). See: https://creativecommons.org/licenses/by-nc-nd/4.0/.

\section{References}

1. Moore A, Donahue T. Pancreatic Cancer. JAMA 2019;322:1426.

2. Rahib L, Smith BD, Aizenberg R, et al. Projecting cancer incidence and deaths to 2030: the unexpected burden of thyroid, liver, and pancreas cancers in the United States. Cancer Res 2014;74:2913-21.

3. Nakao A, Harada A, Nonami T, et al. Lymph node metastasis in carcinoma of the body and tail of the pancreas. Br J Surg 1997;84:1090-2.

4. Versteijne E, Suker M, Groothuis K, et al. Preoperative Chemoradiotherapy Versus Immediate Surgery for Resectable and Borderline Resectable Pancreatic Cancer: Results of the Dutch Randomized Phase III PREOPANC Trial. J Clin Oncol 2020;38:1763-73.

5. Jang JY, Han Y, Lee H, et al. Oncological Benefits of Neoadjuvant Chemoradiation With Gemcitabine Versus Upfront Surgery in Patients With Borderline Resectable Pancreatic Cancer: A Prospective, Randomized, Open-label, Multicenter Phase 2/3 Trial. Ann Surg 2018;268:215-22.

6. Hackert T, Sachsenmaier M, Hinz U, et al. Locally Advanced Pancreatic Cancer: Neoadjuvant Therapy With Folfirinox Results in Resectability in $60 \%$ of the Patients. Ann Surg 2016;264:457-63.

7. Bradley A, Van Der Meer R. Upfront Surgery versus Neoadjuvant Therapy for Resectable Pancreatic Cancer: Systematic Review and Bayesian Network Meta-analysis. Sci Rep 2019;9:4354.

8. Ren X, Wei X, Ding Y, et al. Comparison of neoadjuvant therapy and upfront surgery in resectable pancreatic cancer: a meta-analysis and systematic review. Onco Targets Ther 2019;12:733-44.

9. Unno M, Hata T, Motoi F. Long-term outcome following neoadjuvant therapy for resectable and borderline resectable pancreatic cancer compared to upfront surgery: a meta-analysis of comparative studies by intention-totreat analysis. Surg Today 2019;49:295-9.

10. Versteijne E, Vogel JA, Besselink MG, et al. Meta-analysis comparing upfront surgery with neoadjuvant treatment in patients with resectable or borderline resectable pancreatic cancer. Br J Surg 2018;105:946-58.

11. Ye $M$, Zhang Q, Chen Y, et al. Neoadjuvant chemotherapy for primary resectable pancreatic cancer: a systematic review and meta-analysis. HPB (Oxford) 2020;22:821-32.

12. Gemenetzis G, Groot VP, Blair AB, et al. Survival in Locally Advanced Pancreatic Cancer After Neoadjuvant Therapy and Surgical Resection. Ann Surg 2019;270:340-7.

13. Von Hoff DD, Ervin T, Arena FP, et al. Increased survival in pancreatic cancer with nab-paclitaxel plus gemcitabine. N Engl J Med 2013;369:1691-703.

14. Conroy T, Desseigne F, Ychou M, et al. FOLFIRINOX versus gemcitabine for metastatic pancreatic cancer. $\mathrm{N}$ Engl J Med 2011;364:1817-25.

15. Wolfe AR, Prabhakar D, Yildiz VO, et al. Neoadjuvantmodified FOLFIRINOX vs nab-paclitaxel plus gemcitabine for borderline resectable or locally advanced pancreatic cancer patients who achieved surgical resection. Cancer Med 2020;9:4711-23.

16. Perri G, Prakash L, Qiao W, et al. Response and Survival Associated With First-line FOLFIRINOX vs Gemcitabine and nab-Paclitaxel Chemotherapy for Localized Pancreatic Ductal Adenocarcinoma. JAMA Surg 2020;155:832-9.

17. Macedo FI, Ryon E, Maithel SK, et al. Survival Outcomes Associated With Clinical and Pathological Response Following Neoadjuvant FOLFIRINOX or Gemcitabine/ Nab-Paclitaxel Chemotherapy in Resected Pancreatic Cancer. Ann Surg 2019;270:400-13.

18. Weniger M, Moir J, Damm M, et al. Respect - A multicenter retrospective study on preoperative chemotherapy in locally advanced and borderline resectable pancreatic cancer. Pancreatology 2020;20:1131-8.

19. Dhir M, Zenati MS, Hamad A, et al. FOLFIRINOX Versus Gemcitabine/Nab-Paclitaxel for Neoadjuvant Treatment of Resectable and Borderline Resectable Pancreatic Head Adenocarcinoma. Ann Surg Oncol 2018;25:1896-903.

20. Chapman BC, Gleisner A, Rigg D, et al. Perioperative and Survival Outcomes Following Neoadjuvant FOLFIRINOX versus Gemcitabine Abraxane in Patients with Pancreatic Adenocarcinoma. JOP 2018;19:75-85.

21. Napolitano F, Formisano L, Giardino A, et al. Neoadjuvant Treatment in Locally Advanced Pancreatic Cancer (LAPC) Patients with FOLFIRINOX or Gemcitabine NabPaclitaxel: A Single-Center Experience 
and a Literature Review. Cancers (Basel) 2019;11:981.

22. Gage MM, Wang P, Overton H, et al. Folfirinox is associated with a long-term survival benefit compared to gemcitabine-abraxane for neoadjuvant therapy before pancreatic cancer surgery. HPB 2019;21:S84.

23. Wolfe AR, Miller ED, Abushahin LI, et al. Neoadjuvant FOLFIRINOX versus adjuvant gemcitabine in pancreatic cancer. J Clin Oncol 2019. doi: 10.1200/JCO.2019.37.15suppl.4123.

24. Stroup DF, Berlin JA, Morton SC, et al. Meta-analysis of observational studies in epidemiology: a proposal for reporting. Meta-analysis Of Observational Studies in Epidemiology (MOOSE) group. JAMA 2000;283:2008-12.

25. Stang A. Critical evaluation of the Newcastle-Ottawa scale for the assessment of the quality of nonrandomized studies in meta-analyses. Eur J Epidemiol 2010;25:603-5.

26. Cunningham D, Allum WH, Stenning SP, et al. Perioperative chemotherapy versus surgery alone for resectable gastroesophageal cancer. N Engl J Med 2006;355:11-20.

27. Sauer R, Becker H, Hohenberger W, et al. Preoperative versus postoperative chemoradiotherapy for rectal cancer. N Engl J Med 2004;351:1731-40.

28. van Hagen P, Hulshof MC, van Lanschot JJ, et al. Preoperative chemoradiotherapy for esophageal or junctional cancer. N Engl J Med 2012;366:2074-84.

29. Dhir M, Malhotra GK, Sohal DPS, et al. Neoadjuvant treatment of pancreatic adenocarcinoma: a systematic review and meta-analysis of 5520 patients. World J Surg Oncol 2017;15:183.

30. Zhan HX, Xu JW, Wu D, et al. Neoadjuvant therapy in pancreatic cancer: a systematic review and meta-analysis of prospective studies. Cancer Med 2017;6:1201-19.

31. Cloyd JM, Heh V, Pawlik TM, et al. Neoadjuvant Therapy for Resectable and Borderline Resectable Pancreatic Cancer: A Meta-Analysis of Randomized Controlled Trials. J Clin Med 2020;9:1129.

32. Pusceddu S, Ghidini M, Torchio M, et al. Comparative Effectiveness of Gemcitabine plus Nab-Paclitaxel and FOLFIRINOX in the First-Line Setting of Metastatic Pancreatic Cancer: A Systematic Review and MetaAnalysis. Cancers (Basel) 2019;11:484.

33. Austin PC. Variance estimation when using inverse probability of treatment weighting (IPTW) with survival analysis. Stat Med 2016;35:5642-55.

34. Kobayashi Y, Maeda S, Hama N, et al. Successful conversion surgery for unresectable pancreatic cancer with peritoneal metastases after neoadjuvant albumin-bound paclitaxel and gemcitabine chemotherapy: case report and literature review. Int Cancer Conf J 2017;7:20-5.

35. Nakayasu Y, Shiozawa S, Usui T, et al. A Case of Borderline Resectable Pancreatic Head Cancer Treated by Curative Resection after Chemotherapy. Gan To Kagaku Ryoho 2018;45:2306-8.

36. Kawaguchi K, Motoi F, Yokoyama S, et al. A case of curatively resected locally advanced pancreatic cancer with combined resection of the portal vein and common hepatic artery after neoadjuvant chemotherapy. Gan To Kagaku Ryoho 2013;40:1875-7.

37. Gostimir M, Bennett S, Moyana T, et al. Complete pathological response following neoadjuvant FOLFIRINOX in borderline resectable pancreatic cancer - a case report and review. BMC Cancer 2016;16:786.

38. Luu AM, Hoehn P, Vogel SR, et al. Pathologic Complete Response of Pancreatic Cancer following Neoadjuvant FOLFIRINOX Treatment in Hepatic Metastasized Pancreatic Cancer. Visc Med 2019;35:387-91.

39. Ma T, Bai X, Li G, et al. Neoadjuvant modifiedFOLFIRINOX followed by surgical resection of both the primary and metastatic tumors of a pancreatic hepatoid carcinoma with synchronous liver metastasis: A case report. Medicine (Baltimore) 2017;96:e8413.

40. Valeri S, Borzomati D, Nappo G, et al. Complete pathological response after FOLFIRINOX for locally advanced pancreatic cancer. The beginning of a new era? Case report and review of the literature. Pancreatology 2014;14:425-30.

41. Ahmad SA, Duong M, Sohal DPS, et al. Surgical Outcome Results From SWOG S1505: A Randomized Clinical Trial of mFOLFIRINOX Versus Gemcitabine/ Nab-paclitaxel for Perioperative Treatment of Resectable Pancreatic Ductal Adenocarcinoma. Ann Surg 2020;272:481-6.

42. Conroy T, Hammel P, Hebbar M, et al. FOLFIRINOX or Gemcitabine as Adjuvant Therapy for Pancreatic Cancer. N Engl J Med 2018;379:2395-406.

Cite this article as: Tang R, Meng Q, Wang W, Liang C, Hua J, Xu J, Yu X, Shi S. Head-to-head comparison between FOLFIRINOX and gemcitabine plus nab-paclitaxel in the neoadjuvant chemotherapy of localized pancreatic cancer: a systematic review and meta-analysis. Gland Surg 2021;10(5):15641575. doi: $10.21037 /$ gs-21-16 


\section{Supplementary}

Table S1 The percentage of patients with different resectability across included studies

\begin{tabular}{|c|c|c|c|c|c|c|}
\hline Study & \multicolumn{3}{|c|}{ FOLFIRINOX } & \multicolumn{3}{|c|}{ GA } \\
\hline Wolfe et al., 2020 & $0 \%$ & $67.30 \%$ & $32.70 \%$ & $0 \%$ & $67.30 \%$ & $25 \%$ \\
\hline Macedo et al., 2019 & $21.90 \%$ & $43.20 \%$ & $30.60 \%$ & $23.10 \%$ & $52.70 \%$ & $15.40 \%$ \\
\hline Dhir et al., 2018 & $21 \%$ & $79 \%$ & $0 \%$ & $40.80 \%$ & $59.20 \%$ & $0 \%$ \\
\hline Perri et al., 2020 & $32 \%$ & $31 \%$ & $37 \%$ & $45 \%$ & $23 \%$ & $32 \%$ \\
\hline Weniger et al., 2020 & NA & NA & NA & NA & NA & NA \\
\hline Gage et al., 2019 & NA & NA & NA & NA & NA & NA \\
\hline Napolitano et al., 2019 & NA & NA & NA & NA & NA & NA \\
\hline
\end{tabular}

GA, gemcitabine and nab-paclitaxel; RPC, resectable pancreatic cancer; BRPC, borderline resectable pancreatic cancer; LAPC, locally advanced pancreatic cancer.

Table S2 Compare positive lymph nodes between two groups

Lymph nodes positive

\begin{tabular}{|c|c|c|c|c|}
\hline \multirow[b]{2}{*}{ Study } & \multirow{2}{*}{\multicolumn{2}{|c|}{ FOLFIRINOX }} & & \\
\hline & & & \multicolumn{2}{|c|}{ GA } \\
\hline Wolfe et al., 2020 & $1.3(0-7)$ & & $2.9(0-11)$ & \\
\hline Macedo et al., 2019 & $1(0-2)$ & & $1(0-3)$ & \\
\hline Dhir et al., 2018 & & $56 \%$ & & $71.70 \%$ \\
\hline Perri et al., 2020 & - & - & - & - \\
\hline Weniger et al., 2020 & & $78.20 \%$ & & $51.20 \%$ \\
\hline Gage et al., 2019 & - & - & - & - \\
\hline Napolitano et al., 2019 & - & - & - & - \\
\hline
\end{tabular}

GA, gemcitabine and nab-paclitaxel. 


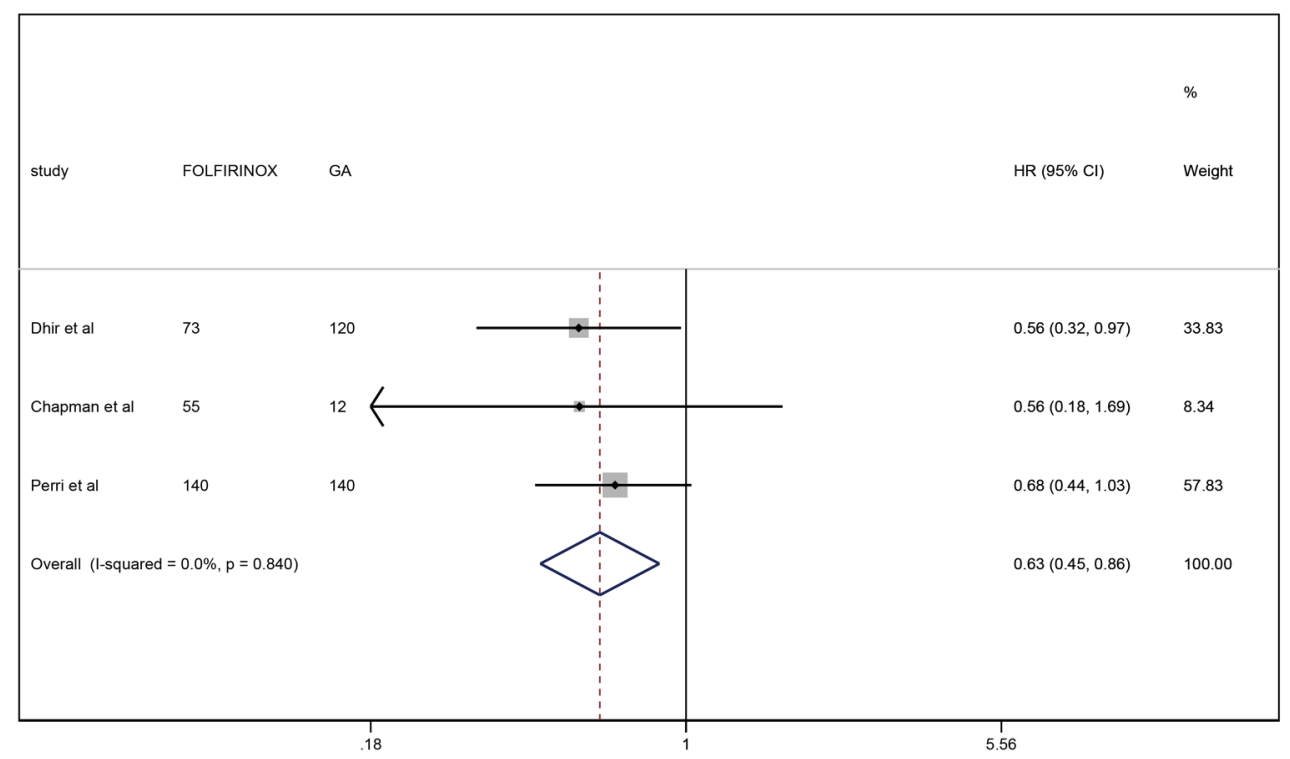

Figure S1 Forest plot showing the confounding factor-adjusted HR for the overall survival interval of patients who received preoperative FOLFIRINOX versus GA.

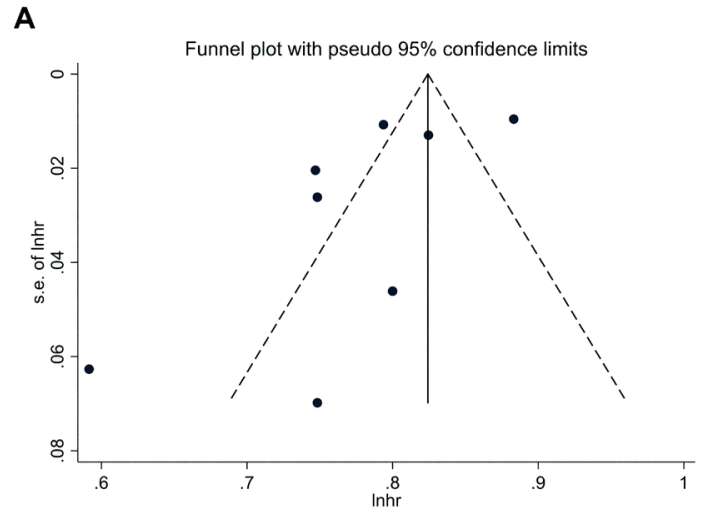

\section{B}

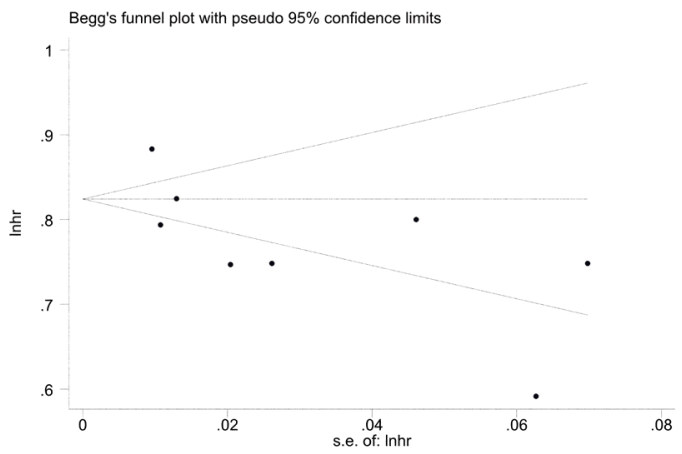

Figure S2 Publication bias detection. (A) Funnel graph. (B) Begg's test. 


\section{A}

FOLFIRINOX

GA

Odds Ratio

Odds Ratio

Study or Subgroup

Events Total Events Total Weight M-H, Fixed, 95\% Cl

M-H, Fixed, 95\% Cl

Chapman et al, 2018

$\begin{array}{lllll}0 & 55 & 1 & 12 & 3.5 \%\end{array}$

$0.07[0.00,1.80]$

Dhir et al, 2018

Gage et al, 2019

$30 \quad 73$

$\begin{array}{lll}37 & 120 & 23.7 \%\end{array}$

Macedo et al, 2019

$37 \quad 105$

$27 \quad 175 \quad 18.9 \%$

$\begin{array}{lllll}74 & 183 & 30 & 91 & 34.3 \%\end{array}$

Weniger, 2020

$32 \quad 103$

$13 \quad 32 \quad 19.7 \%$

$1.57[0.85,2.87]$

$2.98[1.68,5.29]$

$1.38[0.81,2.34]$

$0.66[0.29,1.50]$

Total $(95 \% \mathrm{Cl})$

519

$430 \quad 100.0 \%$

$1.54[1.14,2.08]$

Total events

173

108

Heterogeneity: $\mathrm{Chi}^{2}=12.88, \mathrm{df}=4(\mathrm{P}=0.01) ; \mathrm{I}^{2}=69 \%$

Test for overall effect: $Z=2.82(P=0.005)$

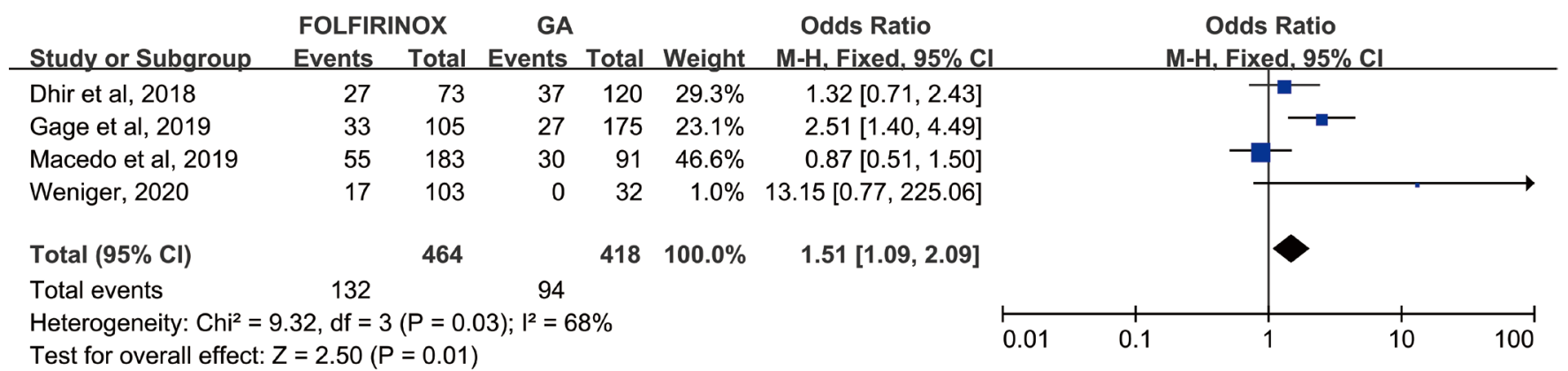

\section{B}

FOLFIRINOX GA

Odds Ratio

Odds Ratio

Study or Subgroup Events Total Events Total Weight $\mathrm{M}-\mathrm{H}$, Random, 95\% Cl

Chapman et al, 2018

$\begin{array}{lllll}0 & 55 & 1 & 12 & 3.1 \%\end{array}$

Dhir et al, 2018

Gage et al, 2019

$\begin{array}{lllll}30 & 73 & 37 & 120 & 24.7 \%\end{array}$

$\begin{array}{lllll}37 & 105 & 27 & 175 & 25.4 \%\end{array}$

Macedo et al, 2019

$74 \quad 183$

$30 \quad 91 \quad 26.3 \%$

Weniger, 2020

$\begin{array}{lllll}32 & 103 \quad 13 \quad 32 \quad 20.5 \%\end{array}$

$0.07[0.00,1.80]$

$1.57[0.85,2.87]$

$2.98[1.68,5.29]$

$1.38[0.81,2.34]$

$0.66[0.29,1.50]$

Total $(95 \% \mathrm{Cl})$

519

$430 \quad 100.0 \%$

Total events

$173 \quad 108$

Heterogeneity: $\mathrm{Tau}^{2}=0.29 ; \mathrm{Chi}^{2}=12.88, \mathrm{df}=4(\mathrm{P}=0.01) ; \mathrm{I}^{2}=69 \%$

Test for overall effect: $Z=0.99(P=0.32)$

$1.36[0.74,2.48]$

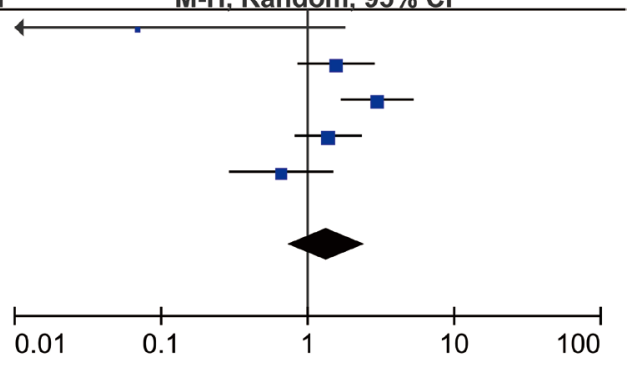

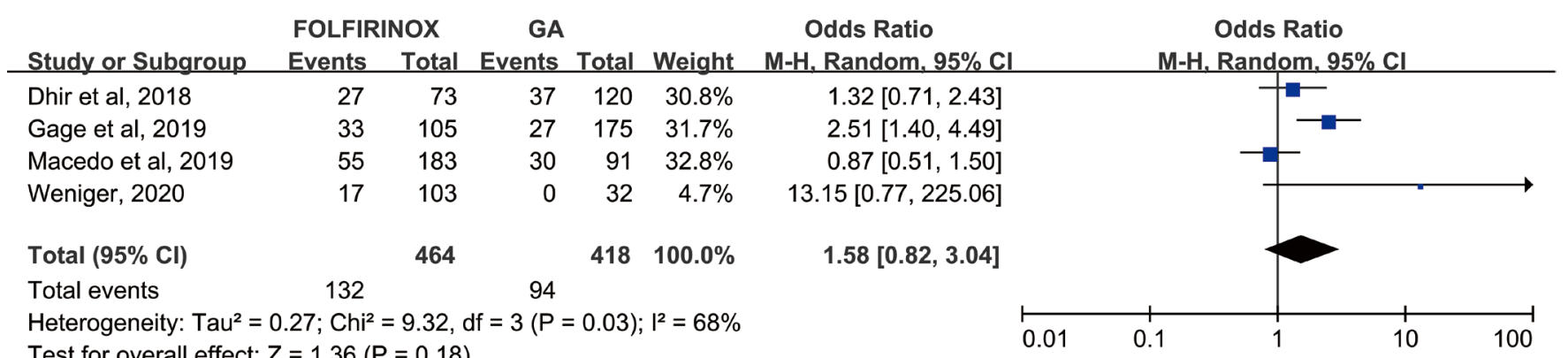

Figure S3 Comparison of four- and five-year survival probability between patients who received preoperative FOLFIRINOX versus GA. (A) Fixed effect model. (B) Random effect model. 
A

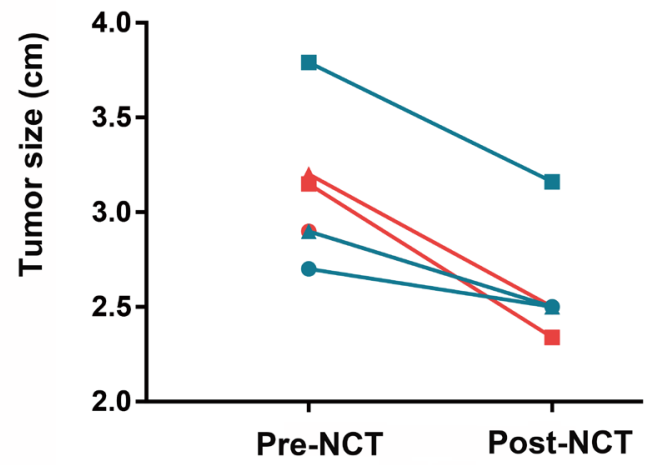

FOLFIRINOX

GA

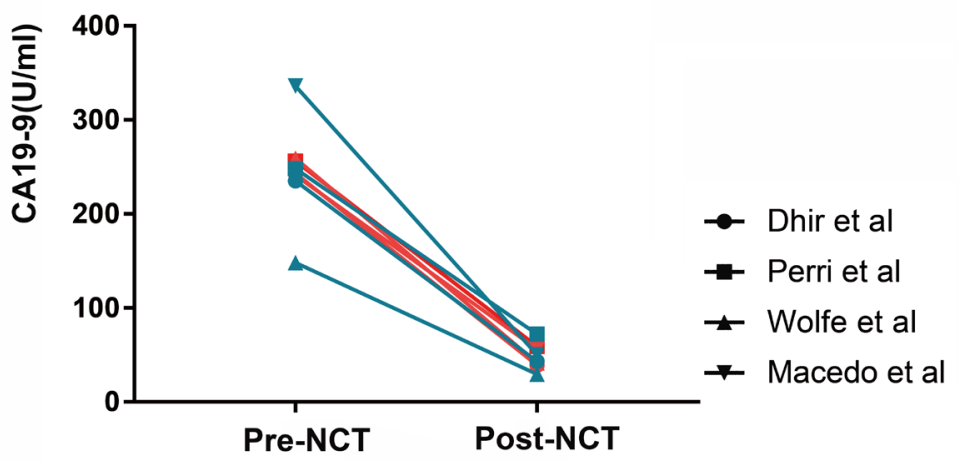

Figure S4 Qualitative analysis presented a similar trend in the variation in (A) tumour size and (B) CA19-9 level between patients who were preoperatively treated with FOLFIRINOX versus GA. 